\title{
Hierarchical Design of Electro-Hydraulic Actuator Control for Vehicle Dynamic Purposes
}

\author{
Balázs Varga, Balázs Németh and Péter Gáspár
}

\begin{abstract}
The paper proposes a hierarchical control design of an electro-hydraulic actuator, which is incorporated in the active anti-roll bars. The aim of the control system is to generate a reference torque, which is required by the vehicle dynamic control. The control-oriented model of the actuator is formulated in two subsystems. The upper-level hydromotor is described in a linear form, while the lower-level spool valve is a polynomial system. These subsystems require different control strategies. At the upper-level a Linear Parameter Varying control is used to guarantee performance specifications. At the lower-level a Control Lyapunov Function-based algorithm, which creates discrete control input values of the valve, is proposed. The interaction between the two subsystems is guaranteed by the spool displacement, which is control input for the upper-level and must be tracked by the lower-level control. The spool displacement has physical constraints, which must also be incorporated into the control design. The operation of the actuator control system is illustrated through a simulation example.
\end{abstract}

\section{INTRODUCTION AND MOTIVATION}

An anti-roll bar is a device which connects the leftand right-hand-side suspensions on an axle. It consists of two torsion bars and a hydraulic actuator. It is essential for vehicles with high center of gravity, such as heavy commercial vehicles. The active anti-roll bar generates a torque between the sprung and unsprung masses in order to improve comfort by reducing body roll and balance the effect of the destabilizing roll moment generated by lateral acceleration.

Various methods in the fields of control design of active anti-roll bars have been presented, see e.g., [1]. An LQG method in the design of anti-roll control was proposed by [2]. A combined differential braking and anti-roll bars with an Linear Parameter Varying (LPV) control design method was proposed by [3]. An active roll control system based on a modified suspension system was developed with distributed control architecture in [4]. A new $H_{\infty} / \mathrm{LPV}$ control method to improve the trade-off between comfort and suspension travel was proposed by [5].

The literature of hydraulic control systems is also very extensive. The robotic applications of the commonly used

B. Varga and B. Németh are with Systems and Control Laboratory, Institute for Computer Science and Control, Hungarian Academy of Sciences, Kende u. 13-17, H-1111 Budapest, Hungary. E-mail: [ bvarga; bnemeth] asztaki.mta.hu

P. Gáspár is with the Institute for Computer Science and Control, Hungarian Academy of Sciences, MTA-BME Control Engineering Research Group, Hungary, E-mail: gaspar.peteresztaki.mta.hu

The research has been conducted as part of the project TÁMOP-4.2.2.A11/1/KONV-2012-0012: Basic research for the development of hybrid and electric vehicles. The Project is supported by the Hungarian Government and co-financed by the European Social Fund. electronically-controlled actuators, such as electromagnetic motors, hydraulic, pneumatic and piezoelectric actuators were detailed and compared in [6]. Several papers have proposed control methods for hydraulic actuators. A nonlinear PID controller for a hydraulic positioning system was proposed by [7]. A velocity tracking robust PID control of a hydraulic cylinder based on linear model with parameter uncertainties was published in [8]. A sliding control to deal with a highly nonlinear model was proposed by [9]. A mathematical model for solenoid valves was proposed by [10]. In [11] a robust low-order control design of an electrohydraulic cylinder was presented and analyzed on a test bed. The modelling and control of a hydraulic servo system, targeting at frequency domain based controller design and the implementation of a LPV controller was proposed by [12]. An LPV modeling and control design with LMI approach for position tracking control of a class of electro-hydraulic servo systems driven by double-rod hydraulic actuators was proposed by [13].

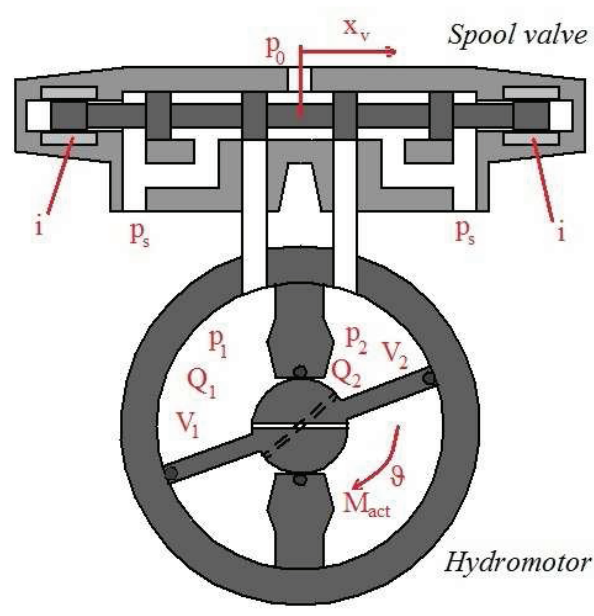

Fig. 1. Oscillating hydromotor actuator

The paper analyses an electro-hydraulic actuator, namely an oscillating hydromotor, controlled by a spool valve. The oscillating hydromotor is a rotary actuator with two cells, separated by vanes. The pressure difference between the vanes generates a torque on the central shaft, which has a limited rotation angle. The hydromotor is connected to a symmetric $4 / 2$ four-way valve and the spool is controlled by a solenoid valve. The spool has a limited distance to move and the input current can only take discrete values. Since the system has high energy density, it requires small space 
and it has low mass. In addition, the actuator has a simple construction, however, it requires an external high pressure pump [14].

In the paper a hierarchical control design of the actuator is proposed. The actuator is modeled as two subsystems, i.e., the hydromotor and the valve. The hydromotor is modelled as a linear system, while an LPV control method is designed to handle physical constraints. The valve is modeled as a polynomial system, while its control design is based on a Control Lyapunov Function. The difficulty in valve control is that its control input may only be discrete values. The advantage of the hierarchical structure is that the hydromotor and the valve are handled in two independent control design steps.

The paper is organized as follows. Section II presents the control-oriented hydromotor- and valve model. Section III describes the interconnection of the two subsystems and the LPV control strategy for the hydromotor. Section IV proposes the controller of the spool valve based on a Control Lyapunov Function. Section V illustrates the operation of the multi-level control system through a simulation example. Finally, Section VI concludes the contributions of the paper.

\section{Modeling the Electro-Hydraulic ACtUAtor}

The hydromotor is modelled as a linear system with a control input $x_{v}$ and a reference $M_{r e f}$. The output of the system is the actuator torque $M_{a c t}$. The pressures in the chambers depend on the flows of the circuits $Q_{1}, Q_{2} \cdot p_{L}$ is the load pressure difference between the two chambers.

The average flow of the system, assuming the supply pressure $p_{s}$ is constant, is as follows:

$$
Q_{L}\left(x_{v}, p_{L}\right)=C_{d} A\left(x_{v}\right) \sqrt{\frac{1}{\rho}\left(p_{s}-\frac{x_{v}}{\left|x_{v}\right|} p_{L}\right)} .
$$

This equation can be linearized around $\left(x_{v, 0} ; p_{L, 0}\right)$ such as

$$
Q_{L}=K_{q} x_{v}-K_{c} p_{L}
$$

where $K_{q}$ is the valve flow gain coefficient and $K_{c}$ is the valve pressure coefficient. In this modeling, the hydromotor does not take into account the friction force and the external leakage flow, see [14]. Moreover, the compressibility of the fluid is considered constant.

The volumetric flow in the chambers is formed as

$$
\dot{p}_{L}=\frac{4 \beta_{E}}{V_{t}}\left(Q_{L}-V_{p} \varphi+c_{l 1} \dot{\varphi}-c_{l 2} p_{L}\right),
$$

where $\beta_{E}$ is the effective bulk modulus, $V_{t}$ is the total volume under pressure and $V_{p}$ is proportional to the areas of vane cross-sections. $c_{l 1}$ and $c_{l 2}$ are parameters of the leakage flow.

The motion equation of the shaft rotation due to the pressure difference $\dot{p}_{L}$ and the external load $M_{r e f}$ is formed as follows:

$$
J \ddot{\phi}=-d_{a} \dot{\varphi}+V_{p} p_{L}+M_{r e f},
$$

where $J$ is the mass of the hydromotor shaft and vanes, $d_{a}$ is the damping constant of the system. Using the principle of momentum, the output torque is

$$
M_{a c t}=A_{v} d_{e} p_{L}
$$

with $A_{v}$ being the area of the vanes and $d_{e}$ is the effective diameter of the vanes. Using (3) and (4) the state-space representation of the hydromotor subsystem is formulated.

The the states of the linear system are the load pressure $p_{l}$, the angular velocity $\dot{\varphi}$ and displacement $\varphi$ of the shaft: $x_{H M}=\left[\begin{array}{lll}p_{L}, & \dot{\varphi}, & \varphi\end{array}\right]^{T}$. The state equation is as follows:

$$
\dot{x}_{H M}=A_{H M} x_{H M}+B_{1 H M} w+B_{2 H M} u,
$$

where the matrices are the following:

$$
\begin{aligned}
& A_{H M}=\left[\begin{array}{ccc}
-\frac{4 \beta_{E}}{V_{t}}\left(c_{2 l}+K_{c}\right) & \frac{4 \beta_{E}}{V_{t}} c_{1 l} & -\frac{4 \beta_{E}}{V_{t}} V_{p} \\
\frac{V_{p}}{J} & -\frac{d_{a}}{J} & 0 \\
0 & 1 & 0
\end{array}\right], \\
& B_{1 H M}=\left[\begin{array}{l}
0 \\
\frac{1}{J} \\
0
\end{array}\right] \\
& B_{2 H M}=\left[\begin{array}{c}
\frac{4 \beta_{E}}{V_{t}} K_{q} \\
0 \\
0
\end{array}\right] \text {. }
\end{aligned}
$$

The measured output is $y_{H M}=M_{a c t}$.

The electronically-controlled spool valve is modeled as a polynomial system, which creates dependence between current $i$ and spool displacement $x_{v}$. In the modeling the nonlinear friction is ignored. The equation of motion is written as follows:

$$
\frac{1}{\omega_{v}^{2}} \ddot{x}_{v}+\frac{2 D_{v}}{\omega_{v}} \dot{x}_{v}+x_{v}=k_{v} \omega_{v}^{2} i
$$

where $k_{v}$ valve gain equals

$$
k_{v}=\frac{Q_{N}}{\sqrt{\Delta p_{N} / 2}} \frac{1}{u_{v \max }},
$$

where $Q_{N}$ is the rated flow at rated pressure and maximum input current, $p_{N}$ is the pressure drop at rated flow and $u_{v \max }$ is the max rated current. $D_{v}$ is the valve damping coefficient, which can be calculated from the apparent damping ratio. $\omega_{v}$ stands for the natural frequency of the valve, see [15].

Let $K_{f}=\omega_{v}^{2}$, which is a spring-stiffness-like parameter. In [14] the flow force stiffness of the system can be approximated as follows:

$$
K_{f}^{\prime} \approx 0.43 w\left(x_{v}\right)\left(p_{s}-p_{L}\right)
$$

where $w\left(x_{v}\right)$ is the area ratio and is a function of $x_{v}$. For control-oriented modeling purposes a polynomial approximation is used for the expression of $K_{f}$.

In the paper $K_{f}$ is approximated by a tenth-order polynomial of $x_{v}$ on the domain $\left[-x_{v, \max },+x_{v, \max }\right]$ :

$$
K_{f}\left(x_{v}\right)=p_{10} x_{v}^{10}+p_{9} x_{v}^{9}+\ldots+p_{1} x_{v}+p_{0},
$$

where $p_{i}$ s are the coefficients of the polynomial. Prior to the control design (7) is rewritten into the following form:

$$
\ddot{x}_{v}=-2 D_{v} \omega_{v} \dot{x_{v}}-K_{f}\left(x_{v}\right) x_{v}+k_{v} \omega_{v}^{2} i .
$$




\section{HIERARCHICAL DESIGN OF ACTUATOR CONTROL}

The actuator is separated into two subsystems, the hydromotor (upper-level) and the valve (lower-level), which are interconnected, see Figure 2. The purpose of the hydromotor control is to track a reference torque $M_{\text {ref }}$ required by the vehicle dynamics. The output signal of the upper-level controller $K_{a c t, u p}$ is a reference spool displacement $x_{v, r e f}$, which must be realized by the valve. The tracking of this reference signal is ensured by the lower-level controller $K_{\text {act,low }}$, which computes discrete values of current $i$ on the solenoids, which cause the displacement of the spool.

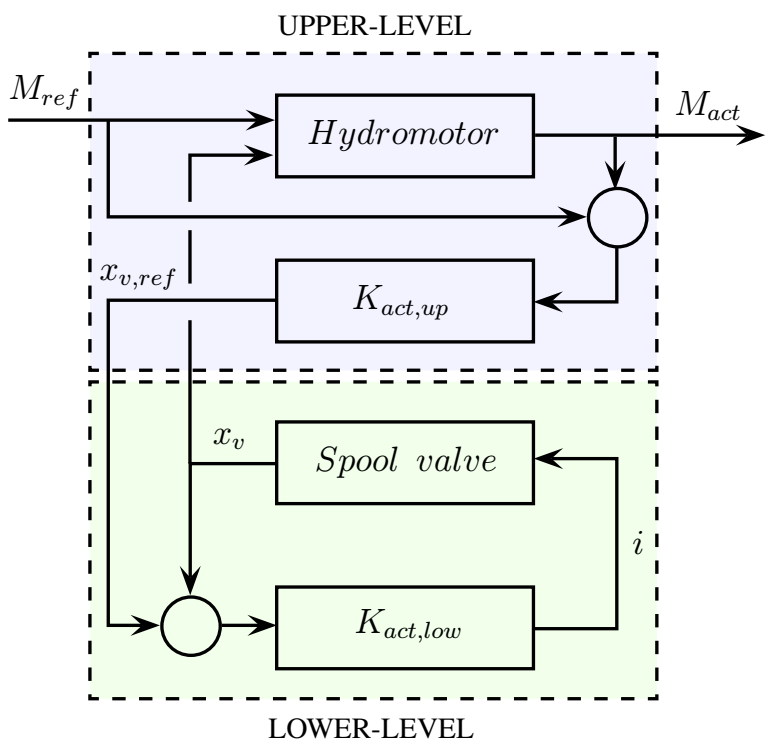

Fig. 2. Architecture of control systems

The two subsystem controls must guarantee different requirements, which require different control approaches. The difficulty in the valve design is that its control input can only be discrete values. The separated modeling also makes it possible to add a double constraint to the model, so the hydromotor controller will never calculate higher reference spool displacements $x_{v, r e f}$ than what the spool can realize. However, there is always a phase difference between the reference signal and the output signal $x_{v}$, therefore the output signal may overshoot and $x_{v}$ may overstep the constraint. To avoid this, there is a constraint for $x_{v}$ in the valve subsystem in the form of a polynomial expression (9). In the independent control design the global stability of the controlled interconnected system must be ensured by the existence of a Common Lyapunov Function. Further details about hierarchical control design are found in [16].

In the following the control design of the upper-level hydromotor is presented. The goal of the control design is to guarantee the tracking of the reference torque $M_{r e f}$ by an appropriate valve motion $x_{v}$. The control input $x_{v}$ is physically realized by the lower-level controlled valve system.

An important factor in hydromotor control is the limited valve displacement $x_{v, s a t}$. Thus, the hydromotor control must guarantee input saturation. In the upper-level actuator control the LPV method is applied, in which the limitation can be incorporated using parameter-dependent weighting functions. Further advantages of the LPV method are its disturbance rejection capability and the guarantee of robustness against unmodelled dynamics.

In the design method two performances are defined which must be guaranteed by the control system such as:

\section{- Torque tracking}

The aim of the control design is to track a reference torque signal $M_{r e f}$, which is defined by the vehicle dynamic control. The performance is formulated as:

$$
z_{1}=M_{\text {ref }}-M_{a c t} ; \quad\left|z_{1}\right| \rightarrow \min
$$

- Valve displacement minimization

Because of limited control input, the valve motion must be handled. The minimization of valve displacement leads to:

$$
z_{2}=x_{v} ; \quad\left|z_{2}\right| \rightarrow \min
$$

In LPV control design several weighting functions are formulated which guarantee a balance between the performances and scale the different signals of the system. Figure 3 illustrates the closed-loop interconnection structure of control design.

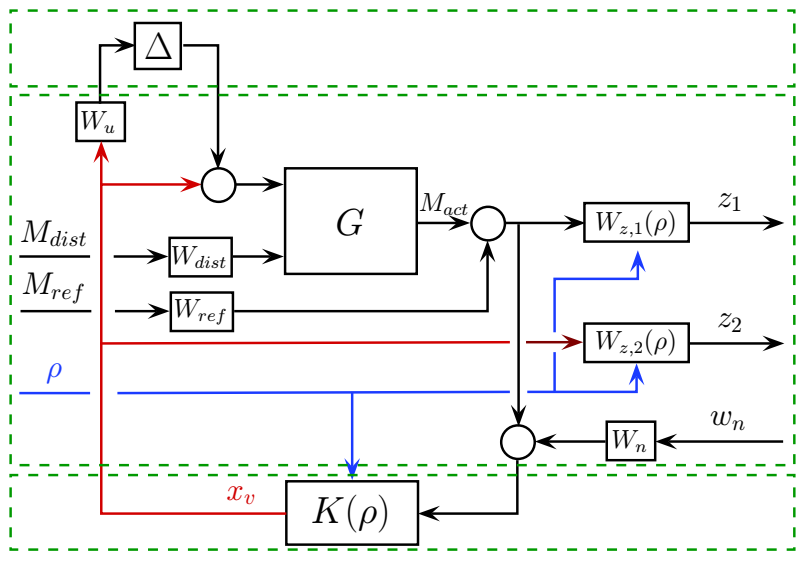

Fig. 3. Closed-loop interconnection structure

The performances $z_{1}, z_{2}$ are considered with parameterdependent weighting functions in the following forms:

$$
\begin{aligned}
& W_{z, 1}=\frac{1}{1-\rho} \cdot \frac{\alpha_{1,1} s+\alpha_{1,0}}{T_{1,1} s+T_{1,0}}, \\
& W_{z, 2}=\frac{1}{\rho} \cdot \frac{\alpha_{2,1} s+\alpha_{2,0}}{T_{2,1} s+T_{2,0}},
\end{aligned}
$$

where $\alpha_{1,1}, \alpha_{1,0}, \alpha_{2,1}, \alpha_{2,0}$ and $T_{1,1}, T_{1,0}, T_{2,1}, T_{2,0}$ are design parameters. In the LPV system the scheduling variable $\rho=\left[\rho_{\min } \ldots \rho_{\max }\right]$ incorporates the information about the actuator saturation.

Figure 4 shows the relationship between $\rho$ and $x_{v}$, where 


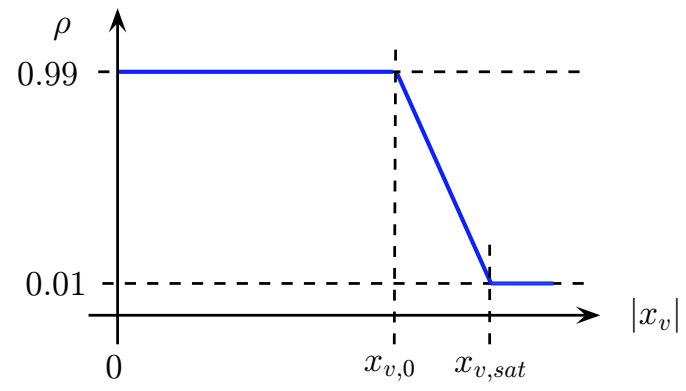

Fig. 4. Scheduling variable $\rho$

$x_{v, 0}$ and $x_{v, \text { sat }}$ are design parameters. If $\rho=0.01$ then $z_{2}$ performance has priority, which leads to the avoidance of $x_{v}>x_{v, \text { sat }}$ signal. However, $z_{1}$ performance is degraded, which results in increased torque tracking error. If $\rho=0.99$ then adequate tracking is achieved, however, it requires larger valve displacement. Consequently, the weighting procedure is the following. If $x_{v}<x_{v, 0}$ then $\rho=\rho_{\max }$, if $x_{v}$ between $x_{v, 0}$ and $x_{v, \text { sat }}$ then

$$
\rho=\rho_{\max } \frac{x_{v, s a t}-x_{v}}{x_{v, s a t}-x_{v, 0}}+\rho_{\min } \frac{x_{v}-x_{v, 0}}{x_{v, s a t}-x_{v, 0}},
$$

and otherwise if $x_{v}>x_{v, \text { sat }}$ then $\rho=\rho_{\min }$. In the method $\rho_{\min }=0.01$ and $\rho_{\max }=0.99$ are selected.

The role of $W_{d i s t}$ and $W_{\text {ref }}$ is to scale the torque disturbance signal $M_{d i s t}$ and reference torque $M_{r e f}$. The control system requires the measurement of tracking error $M_{r e f}-M_{a c t}$. The sensor noise of the measured signal is considered with weighting function $W_{n}$, which gives information about the bound of noise amplitude. $\Delta$ represents the uncertainty on the control input signal, which is derived from the inaccurate realization of $x_{v}$ at the lower-level control.

Based on the closed-loop structure the control problem is formed, in which the robustness against uncertainties and disturbance rejection must be guaranteed. The quadratic LPV performance problem is to choose the parameter-varying controller $K(\rho)$ in such a way that the resulting closed-loop system is quadratically stable and the induced $\mathcal{L}_{2}$ norm from the disturbance and the performances is less than the value $\gamma$ [17], [18]. The minimization task is the following:

$$
\inf _{K} \sup _{\varrho \in \mathcal{F}_{\mathcal{P}}} \sup _{\|w\|_{2} \neq 0, w \in \mathcal{L}_{2}} \frac{\|z\|_{2}}{\|w\|_{2}}
$$

The relation between the state, or output, and the parameter $\rho=\sigma(x)$ is used in the construction of the LPV controller, where $\sigma(x)$ is a measured signal.

\section{Control strategy of the Valve}

The valve control aims to track the reference spool displacement, defined by the controller of the hydromotor. This performance must be satisfied with the shortest settling time possible. Also the control input $i$ can only take three discrete values:

$$
i=\left\{-i_{\max }, i_{0}, i_{\max }\right\}
$$

where $i_{0}=0$. The control strategy is based on the Control Lyapunov Function. It is used to test whether a control input is able to stabilize the system.

Definition 1: Let a dynamical system be given of the form

$$
\dot{x}(t)=f(x(t))+g(x(t)) u,
$$

where $x(t) \in \mathbb{R}^{n}, u(t) \in \mathbb{R}$ and $f$ and $g$ are smooth vector fields and $f(0)=0$. The function $V$ is a Control Lyapunov Function if $V: \mathbb{R}^{n} \rightarrow \mathbb{R}$ is a smooth, radially unbounded and positive definite function.

The existence of such function implies that the system is asymptotically stabilizable at the origin, see [19].

The dynamical system has a differentiable ControlLyapunov Function if and only if there exists a regular stabilizing feedback $\mathrm{u}(\mathrm{x})$. It is called Artstein's theorem.

The tracking error of the control is given as follows:

$$
e=x_{v, r e f}-x_{v} .
$$

The derivative of this expression, assuming that the reference signal is constant for a given interval:

$$
\dot{e}=-\dot{x}_{v}
$$

Define the function $r$ and its derivative:

$$
\begin{aligned}
& r=\dot{e}+\alpha e=-\dot{x_{v}}+\alpha\left(x_{v, r e f}-x_{v}\right), \\
& \dot{r}=\ddot{e}+\alpha \dot{e}=-\ddot{x}_{v}-\alpha \dot{x}_{v},
\end{aligned}
$$

where $\alpha$ is a positive tuning parameter. Let the Lyapunov Function be given in the form

$$
V=\frac{1}{2} r^{2}
$$

This function is positive definite for every $r$. By deriving this function and substituting (19) the following equation is obtained:

$$
\dot{V}=r \dot{r}=\left(-\ddot{x_{v}}-\alpha \dot{x_{v}}\right)\left(-\dot{x_{v}}+\alpha\left(x_{v, r e f}-x_{v}\right)\right)
$$

By substituting the first row of (10) into (21), formally an equation of an ellipsoid for $\dot{x}_{v}$ and $x_{v}$ is obtained. The solution to the equation gives the limit of the controllable regions, wherein the states of the system can exist. The equation is written as follows:

$$
A_{e}{\dot{x_{v}}}^{2}+B_{e} x_{v}^{2}+C_{e} \dot{x_{v}} x_{v}+D_{e} \dot{x_{v}}+E_{e} x_{v}+F_{e}=0
$$

where $A_{e}, B_{e}, C_{e}, D_{e}, E_{e}, F_{e}$ are the coefficients of the ellipsoid which are achieved by rearranging:

$$
\begin{aligned}
& A_{e}=\alpha-2 D_{v} \\
& B_{e}=-K_{f}\left(x_{v}\right)-2 D_{v} \alpha+\alpha^{2} \\
& C_{e}=-K_{f}\left(x_{v}\right) \alpha \\
& D_{e}=k_{v} \omega_{v}^{2} i+2 D_{v} \alpha x_{v, r e f}-\alpha^{2} x_{v, r e f} \\
& E_{e}=K_{f}\left(x_{v}\right) \alpha x_{v, r e f}+k_{v} \omega_{v}^{2} i \alpha \\
& F_{e}=-k_{v} \omega_{v}^{2} i \alpha x_{v, r e f}
\end{aligned}
$$

The parameter $\alpha$ must be tuned so that the system can reach the feasible states with the given control input. Note 
that $A_{e}, B_{e}, C_{e}, D_{e}, E_{e}, F_{e}$ are all functions of $\alpha$ so it has a significant effect on the shape of the set of the controllable regions. To achieve an acceptable performance, the aforementioned parameter must be chosen carefully.

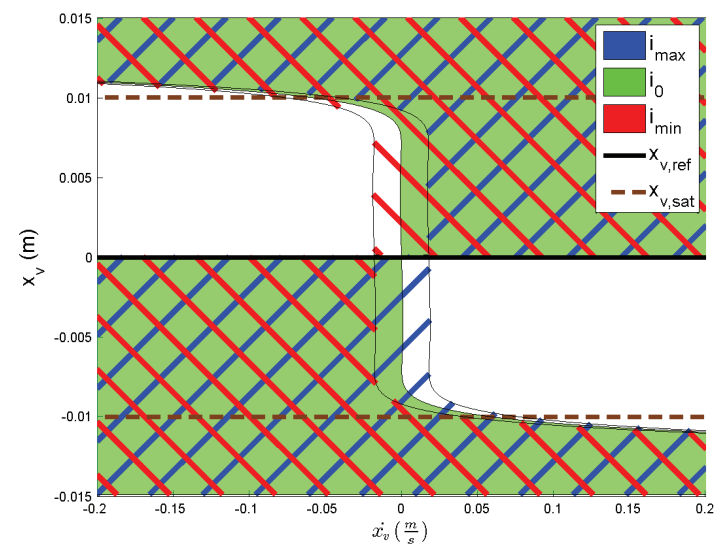

Fig. 5. Controllability regions of the discrete control inputs $\left(x_{v, r e f}=0 m\right)$

The states which can be stabilized by the control input are shown in Figure 5. Since the coefficients in (22) depend on the states, the ellipsoid is degenerated and opened on the $\dot{x}_{v}$, $x_{v}$ plane. The reference signal $x_{v, r e f}$ can only take values between $\pm x_{v, s a t}$, which represent the saturation where the spool of the valve can not open more. The subsets where each control input can stabilize the plant are indicated with different colors.

- There are two domains where none of the control inputs can stabilize the system. However, this does not pose a problem, since the system is stable, see (10).

- There are also domains where multiple inputs can take the system to the reference value. The control strategy exploits this feature to switch between control inputs.

- For different values of $x_{v, r e f}$ the shape of the controllable regions changes, therefore the domain where each control input can operate also changes.

The control algorithm for the spool valve is based on solving the Control Lyapunov Function. For every time step the control strategy calculates the values of the ellipsoids (22) by substituting the momentary values of the states and the reference signal, for each discrete control input. The controller switches between input signals by choosing the appropriate solution. In our strategy the lowest value of the possible solutions is chosen in order to guarantee reference tracking, i.e., $x_{v}$ tends toward $x_{v, r e f}$.

Assuming $E_{\max }, E_{0}, E_{\min }$ are the solution of the ellipsoid equations (22) for $i_{\max }, i_{0}, i_{\min }$ respectively, the control algorithm can be formulated mathematically as follows:

$$
i=\left\{\begin{array}{ccc}
0 & \text { when } & \min \left\{E_{\max }, E_{0}, E_{\min }\right\} \geq 0 \\
i_{\max } & \text { when } & \min \left\{E_{\max }, E_{0}, E_{\min }\right\}=E_{\max } \\
i_{0} & \text { when } & \min \left\{E_{\max }, E_{0}, E_{\text {min }}\right\}=E_{0} \\
i_{\text {min }} & \text { when } & \min \left\{E_{\max }, E_{0}, E_{\text {min }}\right\}=E_{\text {min }}
\end{array}\right.
$$

For energy saving considerations, the control strategy presented above shall be augmented with an additional criterion. If the reference torque on the upper-level $M_{r e f}$ is a predefined small value, the control input is always set at zero. This criterion is necessary because otherwise the output $x_{v}$ would fluctuate around the reference $x_{v, r e f}$, which is zero at this point and the controlled system would never reach equilibrium.

Figure 6 illustrates the domains where each controller can operate at a given reference signal with the given control strategy explained above.

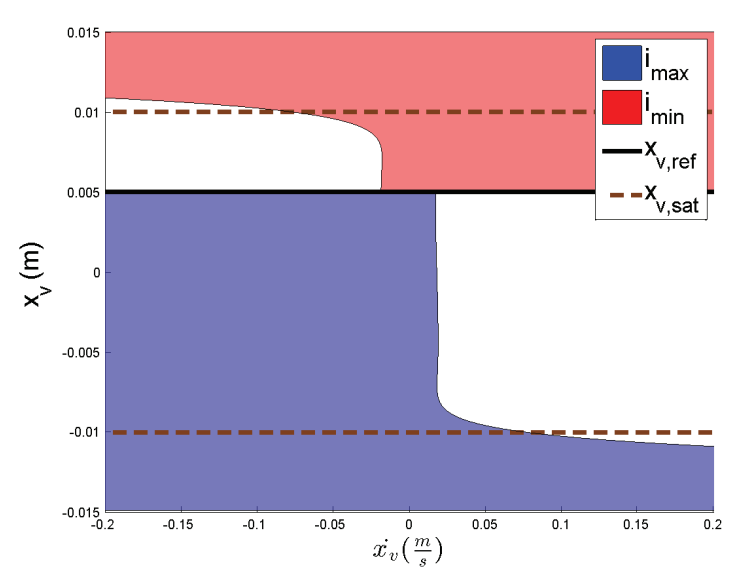

Fig. 6. Control regions of the proposed strategy at $x_{v, r e f}=0.005 m$

\section{Simulation example}

The operation of the electro-hydraulic actuator is presented through a simulation example. In the scenario the parameters of an anti-roll bar actuator of a light vehicle are used. The maximum spool valve displacement is $\left|x_{v, \text { sat }}\right|=10 \mathrm{~mm}$, the discrete current states are $0 A$ and $\pm 0.35 A$.

The input of the system is the reference torque $M_{r e f}$, which is required by the vehicle dynamic control. The torque tracking performance of the actuator is shown in Figure 7(a). Noise on the torque measurement is found in Figure 7(b), which does not have a significant effect on the tracking performances. The undesirable sensor noise can be eliminated by the LPV control.

At high values of the reference torque (eg. at $8 \mathrm{sec}$ and $26 \mathrm{sec}$ ) the tracking error increases because of the limitation of $x_{v}$, see Figure 7(c). In order to avoid the saturation of $x_{v, r e f}$ the scheduling variable $\rho$ is reduced, see Figure 7(d). Thus, the minimization of the valve displacement has a priority, while the tracking error increases. The reference valve position signal $x_{v, \text { ref }}$ computed by the LPV control and the real position $x_{v}$ realized by the lower-level control are illustrated in Figure 7(c).

It is shown that the lower-level controller guarantees the tracking of the reference displacement with an appropriate threshold. Note that the error $x_{v, r e f}-x_{v}$ does not degrade the torque tracking owing to the uncertainty consideration in 
the LPV design. The control current of the valve $i$ is shown in Figure 7(e).

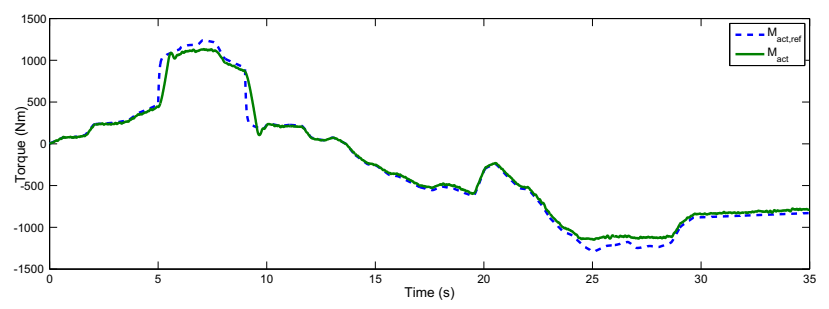

(a) Hydromotor active torque

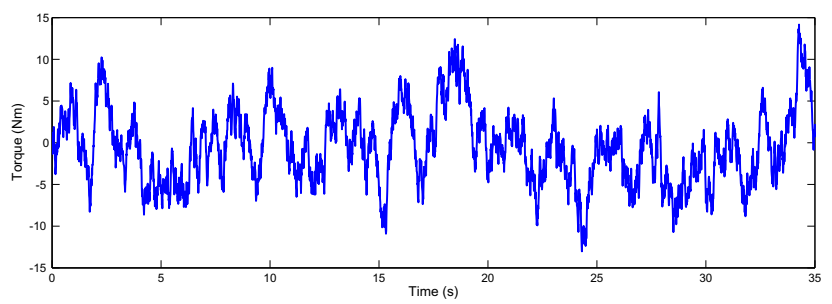

(b) Sensor noise on torque measurement

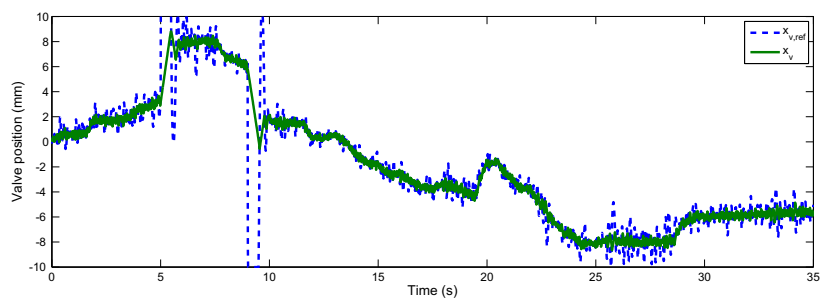

(c) Motion of electrohydraulic valve

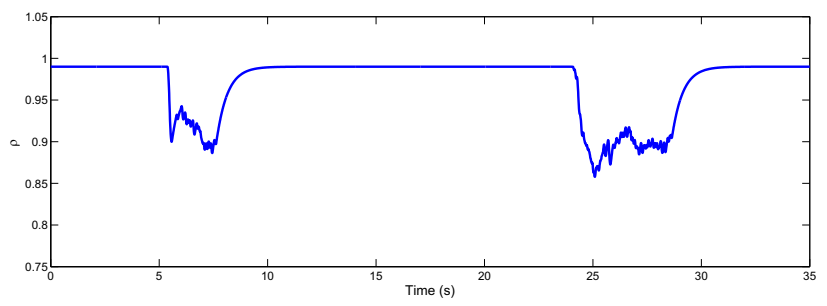

(d) Scheduling variable $\rho$

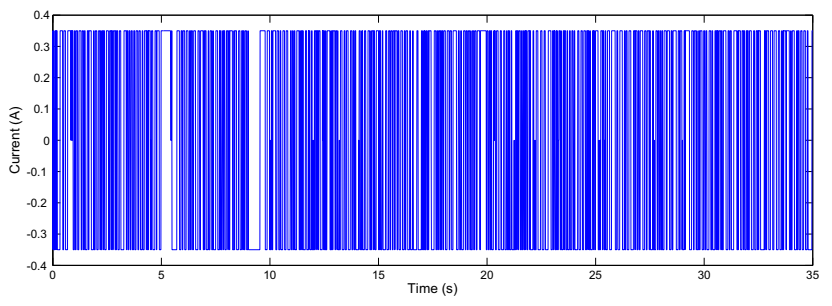

(e) Control current

Fig. 7. Operation of the actuator control

\section{CONCLUSION}

In the paper an electro-hydraulic actuator control design has been proposed. It is based on a hierarchical structure. The actuator is separated into two subsystems. The control of the hydromotor is based on the LPV method and tracks a reference torque while fulfilling a robustness criterion. The valve model has a state constraint for the spool displacement due to physical considerations and the valve control uses the Control Lyapunov Function to calculate discrete input current values.

The simulation results show that the two-step hierarchical actuator control is able to guarantee the tracking of the reference torque signal adequately. Both of the controllers in the hierarchy guarantee the tracking and saturation avoidance capability of the system. Moreover, the controlled system is robust against torque sensor noise and lower-level control imprecision.

\section{REFERENCES}

[1] R. Lin, D. Cebon, and D. Cole, "Optimal roll control of a single-unit lorry," Proceedings of IMechE, Journal of Automobile Engineering, vol. 210 , pp. 44-55, 1996.

[2] D. Sampson and D. Cebon, "Active roll control of single unit heavy road vehicles," Vehicle System Dynamics, vol. 40, pp. 229-270, 2003.

[3] P. Gáspár, I. Szászi, and J. Bokor, "The design of a combined control structure to prevent the rollover of heavy vehicles," European Journal of Control, vol. 10, no. 2, pp. 148-162, 2004.

[4] D. Sampson, G. McKevitt, and D. Cebon, "The development of an active roll control system for heavy vehicles," In Proc. 16th IAVSD Symposium on the Dynamics of Vehicles on Roads and Tracks, Pretoria, South Africa, pp. 704-715, 1999.

[5] A. Do, C. Spelta, S. Savaresi, O. Sename, L. Dugard, and D. Delvecchio, "An lpv control approach for comfort and suspension travel improvements of semi-active suspension systems," Conf. on Decision and Control, 2010.

[6] J. M. Hollerbach, I. W. Hunter, and J. Ballantyne, "A comparative analysis of actuator technologies for robotics," in The robotics review, vol. 2. MIT Press Cambridge, 1992, pp. 299-342.

[7] G. P. Liu and S. Daley, "Optimal-tuning nonlinear PID control of hydraulic systems," Control Engineering Practice, vol. 8, no. 9, pp. 1045-1053, 2000.

[8] M. G. Skarpetis, F. N. Koumboulis, and M. P. Tzamtzi, "Robust control techniques for hydraulic actuators," IEEE Mediterranean Conference on Control and Automation, vol. 15, 2007.

[9] P.-C. Chen and An-Chyauhuan, "Adaptive sliding control of active suspension systems with uncertain hydraulic actuator dynamics," Vehicle System Dynamics, vol. 44, no. 5, pp. 357-368, 2006.

[10] P. Naseradinmousavi and C. Nataraj, "Nonlinear mathematical modeling of butterfly valves driven by solenoid actuators," Applied Mathematical Modeling, vol. 35, pp. 2324-2335, 2011.

[11] N. Niksefat and N. Sepehri, "Design and experimental evaluation of a robust force controller for an electro-hydraulic actuator via quantitative feedback theory," Control Engineering Practice, vol. 8, pp. 1335$1345,2000$.

[12] F. Wijnheijmer, G. Naus, W. Post, M. Steinbuch, and P. Teerhuis, "Modelling and lpv control of an electro-hydraulic servo system," IEEE International Conference on Control Applications, Munich, Germany, 2006.

[13] F. Weng, Y. Ding, and M. Tang, "Lpv model-based robust controller design of electro-hydraulic servo systems," Procedia Engineering, vol. 15, pp. 421-425, 2011.

[14] H. E. Meritt, Hydraulic control systems. John Wiley \& Sons Inc., 1967.

[15] B. Šulc and J. A. Jan, "Non linear modelling and control of hydraulic actuators," Acta Polytechnica, vol. 42, no. 3, pp. 173-182, 2002.

[16] O. Sename, P. Gaspar, and J. Bokor, Robust Control and Linear Parameter Varying Approaches. Springer Verlag, Berlin, 2013.

[17] J. Bokor and G. Balas, "Linear parameter varying systems: A geometric theory and applications," 16th IFAC World Congress, Prague, 2005.

[18] F. Wu, X. Yang, A. Packard, and G. Becker, "Induced $\mathcal{L}_{2}$ norm controller for LPV systems with bounded parameter variation rates," International Journal of Robust and Nonlinear Control, vol. 6, pp. 983-988, 1996.

[19] E. D. Sontag, "A universal construction of Artstein' on nonlinear stabilization," Systems and Control Letters, vol. 13, pp. 117-123, 1989. 